\title{
Cytogenetic findings in patients diagnosed with breast cancer having undergone adjuvant chemotherapy regimens
}

\author{
P.A. Koşar ${ }^{1}$, M. Koçer², D.A. Çelik ${ }^{1}$ and N. Özçelik ${ }^{1}$ \\ 'Department of Medical Biology and Genetics, Süleyman Demirel University, \\ School of Medicine, Isparta, Turkey \\ ${ }^{2}$ Department of Medical Oncology, Süleyman Demirel University, School of Medicine, \\ Isparta, Turkey \\ Corresponding author: P.A. Koşar \\ E-mail: pinarkosar@sdu.edu.tr \\ Genet. Mol. Res. 15 (1): gmr.15017699 \\ Received September 22, 2015 \\ Accepted December 28, 2015 \\ Published March 18, 2016 \\ DOI http://dx.doi.org/10.4238/gmr.15017699
}

\begin{abstract}
The aim of the present study was to investigate DNA damage in peripheral blood lymphocytes of breast cancer (BC) patients before and after administration of chemotherapy. We analyzed the frequency of sister chromatid exchange (SCE), occurrence of micronuclei (MN), and lymphocyte proliferation rate index (PRI) as cytogenetic markers in 28 female BC patients before and after chemotherapy, and in 20 age-matched healthy female volunteers. Prior to treatment, BC patients showed significantly increased background levels of SCE and MN, and lowered PRIs compared to healthy women. In comparison with pre-treatment levels, SCE and MN frequencies were significantly elevated and PRI reduced in blood samples collected after chemotherapy. Our findings indicate that SCE, MN, and PRI may serve as sensitive biomarkers for routine detection of the genetic abnormalities that may occur following administration of antineoplastic drugs in the clinical setting, as well as for the monitoring of high-risk patients receiving chemotherapy for BC.
\end{abstract}

Key words: Breast neoplasms; Chemotherapy; DNA damage; Germline micronucleus; Sister chromatid exchange 


\section{INTRODUCTION}

Breast cancer $(\mathrm{BC})$ is a very common malignancy among women and is second only to lung cancer as the leading cause of cancer-related death (Ely and Vioral, 2007). Moreover, $\mathrm{BC}$ is the most common cancer affecting women. Although epidemiological surveys in Western countries provide a good overview of the incidence and mortality rate associated with this disease, investigations in developing countries are very limited, despite the fact that the incidence of $\mathrm{BC}$ has dramatically increased in recent years (Tazzite et al., 2014). Chemotherapy plays an important role in the management of patients with primary BC. The benefits of adjuvant chemotherapy have been well established in this context (Dignam, 2001; Acevedo et al., 2014), with significant reductions in the risk of relapse and death having been observed in such patients.

However, a significant number of cancer patients develop secondary malignancies unrelated to the original neoplasia that may have arisen due to chemotherapy-induced genetic damage (Carbonell et al., 1996; Elsendoorn et al., 2001). Many types of DNA lesions occur in cells during cancer chemotherapy, which disrupts both neoplastic and non-neoplastic cells, inflicting cytotoxic and mutagenic damage on normal cells.

The frequency of sister chromatid exchange (SCE), the occurrence of micronuclei (MN), and the proliferation rate index (PRI) have been used extensively for cytogenetic examination of peripheral lymphocytes in determining the mutagenic effects of chemotherapeutic drugs (Raposa and Várkonyi, 1987; Digkas et al., 2010). These measures are indicators of exposure to genotoxic chemicals and markers of genome instability and disease states (Carbonell et al., 1996; Kang et al., 1997; Dhillon and Dhillon, 1998; Atalay et al., 2000).

The aim of the present study was to investigate the frequency of SCE and MN and lymphocyte PRI as cytogenetic markers in the peripheral lymphocytes of BC patients before and after adjuvant chemotherapy, and in 20 healthy women with no known family history of BC.

\section{MATERIAL AND METHODS}

A total of 28 non-smoking female BC patients having undergone surgery but having not yet received radiotherapy and chemotherapy, and 20 non-smoking healthy female control subjects residing in the same geographical area (southwest Turkey) were evaluated. The ages of participants were equivalent in the patient and control groups. Control subjects had not received medication for a long period prior to the study and had no family history of BC. Characteristics of the participants are summarized in Table 1. BC patients received intravenously administered chemotherapy according to two main protocols: four cycles anthracycline (AC; 60 $\mathrm{mg} / \mathrm{m}^{2}$ Adriamycin and $600 \mathrm{mg} / \mathrm{m}^{2}$ cyclophosphamide, cycles every 21 days) or four cycles AC (as above) + taxanes. Within the latter group, eight patients received four cycles $A C+12$ weeks paclitaxel $\left(80 \mathrm{mg} / \mathrm{m}^{2}\right.$, cycles every week), while seven patients received four cycles $A C+$ four cycles docetaxel (100 mg/m², cycles every 21 days).

SCE and MN frequency and PRI were determined using cultures of peripheral blood obtained from control (20 women), pre-treatment BC (1 h before administration of first chemotherapy cycle, 28 patients), and post-treatment BC ( $1 \mathrm{~h}$ after completion of chemotherapy cycle; Figures 1 and 2) groups. 
Table 1. Characteristics of breast cancer patients and control subjects.

\begin{tabular}{|c|c|c|}
\hline & Patients [N (\%)] & Controls [N (\%)] \\
\hline Age (years)* & $54.8 \pm 12.5$ & $57.3 \pm 11.5$ \\
\hline \multicolumn{3}{|l|}{ Smoking } \\
\hline Yes & $0(0)$ & $0(0)$ \\
\hline No & $28(100)$ & $20(100)$ \\
\hline \multicolumn{3}{|l|}{ Menopause status } \\
\hline Pre-menopause & $10(35.71)$ & $6(30)$ \\
\hline Post-menopause & $18(64.29)$ & $14(70)$ \\
\hline \multicolumn{3}{|l|}{ Tumor localization } \\
\hline Right breast & $18(64.29)$ & $0(0)$ \\
\hline Left breast & $10(35.71)$ & $0(0)$ \\
\hline \multicolumn{3}{|l|}{ Pregnancy at term } \\
\hline Yes & $24(85.71)$ & $18(90)$ \\
\hline No & $4(14.29)$ & $2(10)$ \\
\hline Numberof pregnancies* & $1.68 \pm 0.2$ & $1.95 \pm 0.35$ \\
\hline
\end{tabular}

*Data are reported as means \pm standard deviation.

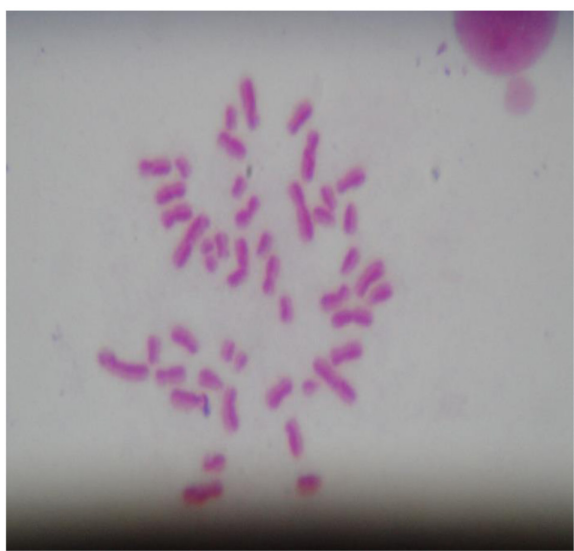

Figure 1. Metaphase samples showing sister chromatid exchange. Samples were taken from the patient group before (A) and after (B) the treatment period.

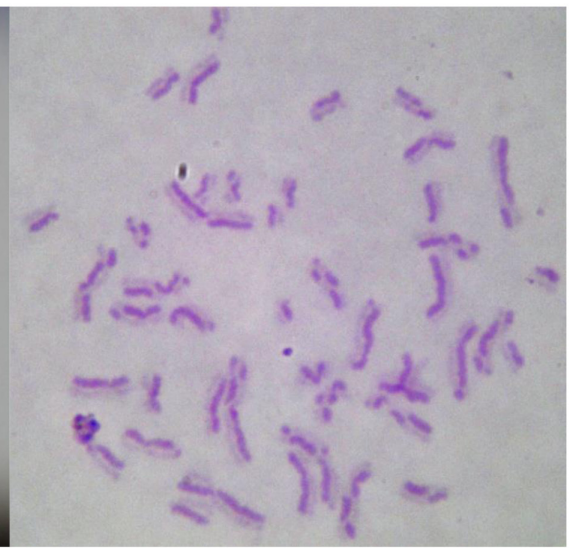

B 
All subjects were informed of the aim and experimental details of the study and gave their informed consent for voluntarily participation. This investigation was also approved by the relevant research Ethics Committee.

\section{Blood sampling}

Peripheral blood was collected from patients before and after chemotherapy treatment and from age- and gender-matched healthy donors of a similar socio-economic status from the same region of Turkey. None of the participants reported a history of alcohol consumption, smoking, treatment with genotoxic medicines, genetic disorders, or any kind of cancer.

Blood culture, harvesting, and slide preparation were carried out according to an established protocol (Barch et al., 1991), with some modifications. Heparinized whole blood was cultured in 4.7 mL RPMI1640 medium (BIO1-106-1B; Biological Industries, Cromwell, CT, USA) containing 20\% fetal calf serum (BIO4-001-1B; Biological Industries), 2\% phytohemagglutinin (BIO12-006-1H; Biological Industries), L-glutamine (BIO3-020-1B Biological Industries), and penicillin/streptomycin (BIO3-0311B, Biological Industries). After 48 h of culture, 5-bromo-2'-deoxyuridine (BrdU; B5002; Sigma-Aldrich, St. Louis, MO, USA) was added at a final concentration of $10 \mu \mathrm{g} / \mathrm{mL}$. Cultures were established in darkly stained flasks around which aluminum foil had been wrapped to protect them from light. Total culture time was $72 \mathrm{~h}$, including treatment with $10 \mu \mathrm{g} / \mathrm{mL}$ colcemid (12-003-1C; Biological Industries) for the final $2 \mathrm{~h}$. Cells were then exposed to a hypotonic solution of potassium chloride at $37^{\circ} \mathrm{C}$ for 10 min before being fixed in a methanol:glacial acetic acid (3:1) mixture and thoroughly washed in fresh fixative approximately three times. SCE staining was performed as previously described (Perry and Wolff, 1974). Slides were stained with Giemsa stain in phosphate buffer, pH 7.0.

For MN analysis, whole-blood cultures were established as above, but without addition of BrdU. To obtain binucleated cells, $6 \mu \mathrm{g} / \mathrm{mL}$ cytochalasin B was added to each culture after $68 \mathrm{~h}$. In all other respects, the procedure was the same as that used for measurement of SCE. Under 100X magnification, at least 1000 binucleate cells with well-preserved cytoplasms were scored for each patient. Micronucleus frequency was determined by counting the total number of MN in all binucleate cells and dividing this by the total number of such cells.

For analysis of lymphocyte PRI, fluorescence plus Giemsa-stained preparations were used, in which cells dividing for the first (M1), second (M2), or third time (M3) in a BrdU-containing culture were identified by differential sister chromatid staining. Briefly, M1 cells contained chromosomes with uniformly darkly-stained sister chromatids. M2 cells showed only harlequin chromosomes, each consisting of one darkly- and one lightly-stained chromatid, whereas M3 cells contained chromosomes in which both sister chromatids were uniformly lightly stained. Lymphocyte proliferation kinetics were studied on 100 differentially stained metaphases per blood sample. The PRI was calculated according to the formula: $\mathrm{PRI}=(\mathrm{M} 1+2 \mathrm{M} 2+3 \mathrm{M} 3) / 100$, as reported by Lamberti et al. (1983).

\section{Statistical analyses}

Statistical analysis was performed with the GraphPad InStat program (version 3; GraphPad Software, Inc., La Jolla, CA, USA). The Mann-Whitney U-test and Wilcoxon matched-pairs test were used for interpretation of results where appropriate. The level of statistical significance was defined as 0.05 .

\section{RESULTS}

None of the study subjects were smokers, and the mean ages of the patient and control 
groups were similar (54.8 \pm 12.5 and $57.3 \pm 11.5$ years, respectively, $P=0.4265$; Table 1$)$. The clinical and pathological characteristics of patients were taken into account in the analysis of SCE, $\mathrm{MN}$, and PRI measurements. The mean values of these measures with respect to tumor stage and grade, lymph node involvement, histologic tumor type, and hormone receptor and c-ERBB2 status are shown in Table 2. Tumor stage, histologic type, and receptor and c-ERBB2 status had no effect on SCE, MN, or PRI figures.

\begin{tabular}{|c|c|c|c|c|}
\hline Feature & Patients [N (\%)] & $\mathrm{SCE}^{*}$ & $\mathrm{MN}^{*}$ & $\mathrm{PRI}^{*}$ \\
\hline \multicolumn{5}{|l|}{ Tumor stage } \\
\hline T1 & $5(33.3)$ & $10.8 \pm 2.8$ & $6.6 \pm 2.2$ & $1.63 \pm 0.1$ \\
\hline $\mathrm{T} 2$ & $14(43.3)$ & $11.4 \pm 2.4$ & $9.6 \pm 4.5$ & $1.61 \pm 0.1$ \\
\hline T3-4 & 9 (23.3) & $11 \pm 1.2$ & $11.8 \pm 4.6$ & $1.57 \pm 0.2$ \\
\hline$P$ value & & 0.988 & 0.051 & 0.174 \\
\hline \multicolumn{5}{|c|}{ Lymph nodeinvolvement } \\
\hline Positive & $19(67.9)$ & $11.3 \pm 2.3$ & $10.5 \pm 4.8$ & $1.59 \pm 0.14$ \\
\hline Negative & $9(32.1)$ & $10.8 \pm 1.7$ & $8.2 \pm 3.4$ & $1.61 \pm 0.09$ \\
\hline$P$ value & & 0.902 & 0.227 & 0.445 \\
\hline \multicolumn{5}{|l|}{ Histologic type } \\
\hline Invasive ductal & $19(67.9)$ & $11.2 \pm 1.5$ & $10.2 \pm 4.1$ & $1.59 \pm 0.14$ \\
\hline Invasive lobular & $7(25)$ & $12.1 \pm 3$ & $10.6 \pm 5.5$ & $1.61 \pm 0.11$ \\
\hline$P$ value & & 0.739 & 0.915 & 0.716 \\
\hline \multicolumn{5}{|l|}{ Estrogen receptor } \\
\hline Positive & $20(71.43)$ & $11 \pm 1.5$ & $9.8 \pm 4.2$ & $1.59 \pm 0.1$ \\
\hline Negative & $8(28.57)$ & $11.6 \pm 3.2$ & $9.8 \pm 5.4$ & $1.62 \pm 0.2$ \\
\hline$P$ value & & 0.819 & 0.779 & 0.919 \\
\hline \multicolumn{5}{|c|}{ Progesterone receptor } \\
\hline Positive & $18(62.29)$ & $10.8 \pm 1.5$ & $10.1 \pm 4.3$ & $1.6 \pm 0.1$ \\
\hline Negative & $10(35.71)$ & $11.8 \pm 2.9$ & $9.3 \pm 4.9$ & $1.58 \pm 0.2$ \\
\hline $\mathrm{P}$ value & & 0.632 & 0.533 & 0.231 \\
\hline \multicolumn{5}{|l|}{ c-ERBB2 } \\
\hline Positive & $22(78.57)$ & $11 \pm 2.1$ & $10 \pm 4.9$ & $1.60 \pm 0.13$ \\
\hline Negative & $6(21.43)$ & $11.7 \pm 2.3$ & $9 \pm 2.3$ & $1.62 \pm 0.1$ \\
\hline$P$ value & & 0.1572 & 0.8665 & 0.6542 \\
\hline
\end{tabular}

*Data are reported as means \pm standard deviation.

The PRI and frequency of SCE and MN in lymphocytes from control subjects and preand post-chemotherapy BC patients are summarized in Table 3. Mean SCE and MN frequencies were significantly higher in BC patients compared to controls $(P<0.0001$ for both). These values also significantly increased following chemotherapy $(P<0.0001$ compared to pre-treatment levels, for both). Mean PRI was significantly lower in patients compared to controls $(P<0.0001)$, and decreased statistically significantly after chemotherapy in the patient group $(P<0.0001$ compared to pre-chemotherapy levels; Table 3).

Table 3. Sister chromatid exchange (SCE), micronuclei (MN), and proliferation rate index (PRI) values in breast cancer patients before and after chemotherapy and control subjects.

\begin{tabular}{|c|c|c|c|}
\hline Group (N) & $\mathrm{SCE}^{*}$ & $\mathrm{MN}^{*}$ & $\mathrm{PRI}^{*}$ \\
\hline Pre-chemotherapy (28) & $11.2 \pm 2.1(7.6-18.5)^{\mathrm{a}, \mathrm{d}}$ & $9.8 \pm 4.5(5-22)^{b, e}$ & $1.60 \pm 0.1(1.36-1.92)^{c, f}$ \\
\hline Post-chemotherapy (28) & $14.1 \pm 3.9(7.9-30.2)$ & $15.4 \pm 5.4(6-28)$ & $1.35 \pm 0.2(0.96-1.67)$ \\
\hline Control (20) & $7.4 \pm 2.6(3.2-11.8)$ & $3.9 \pm 1.8(1-8)$ & $1.82 \pm 0.2(1.6-2.16)$ \\
\hline
\end{tabular}


Concerning the chemotherapy protocols used, the AC + taxanes group demonstrated higher mean SCE and MN frequencies and lower PRI values than the AC group. However, only MN measurements differed significantly in response to the AC + taxanesprotocol (Table 4). Mean SCE, $\mathrm{MN}$, and PRI values did not statistically differ between the two AC + taxanes subgroups (Table 4).

Table 4. Sister chromatid exchange (SCE), micronuclei (MN), and proliferation rate index (PRI) measurements from breast cancer patients under various chemotherapy protocols.

\begin{tabular}{|c|c|c|c|}
\hline Protocol (N) & SCE $^{*}$ & $\mathrm{MN}^{*}$ & $\mathrm{PRI}^{*}$ \\
\hline Anthracycline (13) & $13.48 \pm 2.73$ & $13.46 \pm 5.14$ & $1.39 \pm 0.17$ \\
\hline Anthracycline + taxanes (15) & $14.7 \pm 4.64$ & $17.4 \pm 4.64^{a}$ & $1.31 \pm 0.19$ \\
\hline$P$ value & 0.8538 & 0.0474 & 0.2493 \\
\hline \multicolumn{4}{|l|}{ Anthracycline+taxanes subgroups } \\
\hline Anthracycline+docetaxel (7) & $14.19 \pm 2.02$ & $17.29 \pm 3.99$ & $1.42 \pm 0.12$ \\
\hline Anthracycline + paclitaxel (8) & $15.72 \pm 6.01$ & $17.75 \pm 5.4$ & $1.26 \pm 0.22$ \\
\hline$P$ value & 0.9999 & 0.7278 & 0.1320 \\
\hline
\end{tabular}

${ }^{*}$ Data are reported as means \pm standard deviation. ${ }^{a} \mathrm{P}=0.0474$, anthracycline $v s$ anthracycline + taxanes.

Patients were followed-up for a mean of $38.89 \pm 6.35$ months after administration of chemotherapy. While 21 of 28 subjects did not develop metastasis, seven patients did, four of whom died as a consequence during the follow-up period. Mean SCE, MN, and PRI values following chemotherapy administration were compared in patients according to their metastasis status. Although a trend towards increased cytogenetic damage in patients without metastasis was observed, the values involved did not significantly differ between the two groups (Table 5).

Table 5. Sister chromatid exchange (SCE), micronuclei (MN), and proliferation rate index (PRI) values of breast cancer patients according to metastasis status during the follow-up period.

\begin{tabular}{l|c|c|c}
\hline Group (N) & SCE $^{*}$ & $\mathrm{MN}^{*}$ & $\mathrm{PRI}^{*}$ \\
\hline No metastasis (21) & $14.45 \pm 3.99$ & $16.14 \pm 4.94$ & $1.32 \pm 0.19$ \\
\hline Metastasis (7) & $13.18 \pm 3.48$ & $13.86 \pm 5.92$ & $1.43 \pm 0.15$ \\
\hline P value & 0.6907 & 0.3394 & 0.1176 \\
\hline
\end{tabular}

*Data are reported as means \pm standard deviation.

\section{DISCUSSION}

In the present study, the extent of spontaneous chromosome damage in lymphocytes of $\mathrm{BC}$ patients and control subjects was examined. Basal levels of DNA damage in peripheral lymphocytes of untreated BC patients have been described previously (Cefle et al., 2006; Kopjar et al., 2007; Aristei et al., 2009; Santos et al., 2010; Çelik et al., 2013). In these studies, such damage was found to be significantly higher in BC patients than in control subjects. Our results are consistent with these findings (Table 3). We used PRI and SCE and MN frequency as markers of DNA modifications in peripheral blood lymphocytes of BC patients, finding all these measures to be sensitive gauges of damage. We observed an elevated background incidence of SCE and MN and altered proliferation kinetics in such cells in BC patients, suggesting increased susceptibility to genomic damage compared with healthy women.

Clinical, histologic, and biological factors such as grade, stage, and hormone receptor and c-ERBB2 status have an impact on tumor aggressiveness, response to chemotherapy, and survival in BC patients (Shet et al., 2007; Traoré et al., 2012; Han et al., 2015). The influence of these clinicopathological features on DNA damage in BC patients has been examined in a prior study (Santos et al., 2010). Tumor stage, hormone receptor status, c-ERBB2 protein expression, and 
lymph node involvement were observed to have no effect on levels of spontaneous DNA damage in lymphocytes of untreated BC patients. Similarly, neither these factors nor tumor histologic type significantly influenced SCE, MN, and PRI values among untreated BC patients in the present study (Table 2). However, owing to the relatively small number of patients studied, these results require further investigation using larger patient groups.

At the time of intravenous chemotherapy application, most normal cells are in quiescent states, while most cancer cells are actively proliferating. Although the goal of chemotherapy is to remove the latter while sparing the former, some adverse effects on normal cells almost always occur. If the level of DNA damage induced by chemotherapy in non-target cells is too high, the affected cells will usually die by apoptosis. In surviving cells, unrepaired DNA will contribute to the appearance of SCE and MN, while the cytostatic effects of chemotherapy will lower the PRI. It is thought that the extent of genetic damage in surrogate cells, such as peripheral lymphocytes, correlates with similar events in the targeted tissues (Mourelatos, 1996). Studies have revealed structural chromosomal damage in peripheral blood lymphocytes after chemotherapy in patients with various types of cancer (Xu et al., 1983; Bilban-Jakopin, 2000; Bilban-Jakopin and Bilban, 2001). In the present study, the administration of chemotherapy was found to induce genotoxic damage in non-target cells of BC patients (Table 3). Following such intravenous treatment, the frequency of SCE and MN in the peripheral blood lymphocytes of these patients increased with respect to pre-chemotherapy levels ( $P<0.0001$, for both). Moreover, in comparison to pre-treatment specimens, post-treatment blood samples showed significantly reduced $P R I$ values $(P<0.0001$; Table 3). Similar results have been obtained by others (Kopjar et al., 2007; Tekcan et al., 2012).

The nature of the damage induced by in vivo exposure to chemotherapy is somewhat complex. Studies of genotoxic damage in patients treated with multiple cytotoxic drugs are difficult to interpret, as the contribution of each drug to the overall effect cannot be ascertained. Due to their divergent modes of action, the various classes of antineoplastic drugs are expected to cause different types of DNA lesions, and to varying extents. Other authors have also investigated the levels of DNA damage in peripheral blood lymphocytes of BC patients after chemotherapy (Kopjar et al., 2007; Tekcan et al., 2012). For instance, Kopjar et al. (2007) recorded a significantly elevated SCE frequency and lowered PRI in peripheral blood samples collected following treatment. Patients received chemotherapy according to three standard protocols: cyclophosphamide + methotrexate + 5-fluorouracil; 5-fluorouracil + Adriamycin + cyclophosphamide (FAC); and AC, consisting of Adriamycin + cyclophosphamide. The FAC protocol was shown to be the most genotoxic of those studied (Kopjar et al., 2007). Tekcan et al. (2012) also recorded a significantly augmented SCE rate in post-chemotherapy peripheral blood lymphocytes. In their investigation, patient groups were treated with AC, 5-fluorouracil + epirubicin + cyclophosphamide, or epirubicin + cyclophosphamide. However, no significant differences between these groups were observed in terms of SCE measurements.

Recommendations regarding the use of adjuvant chemotherapy for $\mathrm{BC}$ are based on risk to the individual patient and the balance between absolute benefit and toxicity. Anthracyclinebased regimens are preferred, and addition of taxanes has been shown to increase survival rate (Gonzalez-Angulo et al., 2007). In the present study, all patients were treated with intravenously administered Adriamycin and cyclophosphamide, with some receiving paclitaxel or docetaxel in addition. The highest level of chromosomal damage was observed in patients having been treated with AC + taxanes protocols (Table 4). Tekcanet al. (2012) previously demonstrated that the number of drugs used in a regimen has no effect on the level of DNA damage incurred. However, the increase in genetic abnormalities observed in our AC + taxanes group is not necessarily at odds 
with this finding. This result may not have been caused by the number of chemotherapeutic agents used, but rather suggests that particular drugs in an adjuvant chemotherapy protocol have greater effects than others on genetic integrity. Nevertheless, this may also be explained by an imbalance in the dosage of drugs used in adjuvant therapy, or many other factors. Further investigation is required to elucidate the precise mechanism involved.

The carcinogenic effects of antineoplastic drugs appear to be cumulative, and the number of chemotherapy cycles is a strong determinant of secondary cancer risk in patients. Levels of genotoxic damage in peripheral blood lymphocytes might reflect those sustained by other cells or tissues. Studies assessing the relationship between cytogenetic alterations in cancer patients after therapy and later onset of secondary tumors would be important in judging whether these biomarkers can be used to predict the long-term consequences of certain regimens, and thus influence the choice of treatment modality. Tekcan et al. (2012) showed that an increase in the number of chemotherapy cycles causes greater genome instability. Patients in their study underwent intravenous administration of two to six chemotherapy cycles. Higher numbers of cycles were found to increase genotoxic alterations. In the present investigation, patients underwent four cycles of $A C$ or four cycles of $A C+$ four cycles of docetaxel or 12 weeks of paclitaxel. The highest level of DNA damage was observed following administration of $A C+$ taxane regimens (Table 4). Although the effect of cycle number on genome alteration appears to be important, analyzing our current data in this respect is not feasible owing to the particular chemotherapy protocols used.

Concerning its response to chemotherapy, BC is a highly heterogeneous malignancy (Han et al., 2015). Histopathology, enzyme polymorphisms (including those affecting glutathione S-transferases), and clinical features, including tumor grade and stage, hormone receptor status, and c-ERBB2 expression, are used to predict response to chemotherapy in BC patients (Colleoni et al., 2010; Han et al., 2015; Wang et al., 2015). Although many of these factors have been shown to be imprecise in anticipating treatment outcome, it is not known whether genetic markers such as SCE, $\mathrm{MN}$, and PRI affect clinical results in BC. We have provided data concerning the relevance of changes in these measures to patient outcomes. Seven study participants developed metastasis during a mean follow-up period of $38.89 \pm 6.35$ months, during which four patients died. DNA damage measurements did not significantly differ between patients with stable conditions and those with metastatic cancer (Table 5). Further investigations involving large patient groups may revise this finding, and provide more informative results than those achieved with the relatively small number of patients in this study.

To conclude, this study proposes SCE and MN frequency and PRI to besensitive biomarkers for detection of the acute cytogenetic effects of antineoplastic drugs in peripheral blood lymphocytes of BC patients. These tests may be used for the cytogenetic biomonitoring of cancer patients. A more meaningful scientific interpretation of our results requires larger patient populations, and comprehensive and detailed investigations into the genotoxic side-effects of chemotherapeutic drugs.

\section{Conflicts of interest}

The authors declare no conflict of interest.

\section{REFERENCES}

Acevedo F, Sanchez C, Jans J, Rivera S, et al. (2014). How much survival benefit is necessary for breast cancer patients to opt for adjuvant chemotherapy? Results from a Chilean survey. Ecancermedicalscience 8: 391. 
Aristei C, Stracci F, Guerrieri P, Anselmo P, et al. (2009). Frequency of sister chromatid exchanges and micronuclei monitored over time in patients with early-stage breast cancer: results of an observational study. Cancer Genet. Cytogenet. 192: 24-29. http://dx.doi.org/10.1016/j.cancergencyto.2009.02.019

Atalay F, Baltaci V, Alpas I, Savas I, et al. (2000). Sister chromatid exchange rate from pleural fluid cells in patients with malignant mesothelioma. Mutat. Res. 465: 159-163. http://dx.doi.org/10.1016/S1383-5718(99)00224-7

Barch MJ, Lawce HJ and Arsham MS (1991). Peripheral blood culture. In: The ACT Cytogenetics Laboratory Manual (Barch MJ, ed.). 2nd edn. Raven Press, New York.

Bilban-Jakopin C (2000). Chromosomal changes in somatic cells in seminoma patients after treatment with ionizing radiation or cytostatics. Neoplasma 47: 48-55.

Bilban-Jakopin C and Bilban M (2001). Genotoxic effects of radiotherapy and chemotherapy on circulating lymphocytes in patients with Hodgkin's disease. Mutat. Res. 497: 81-88. http://dx.doi.org/10.1016/S1383-5718(01)00237-6

Carbonell E, Demopoulos NA, Stefanou G, Psaraki K, et al. (1996). Cytogenetic analysis in peripheral lymphocytes of cancer patients treated with cytostatic drugs: results from an EC Collaborative Study. Anticancer Drugs 7: 514-519. http://dx.doi. org/10.1097/00001813-199607000-00003

Cefle K, Ucur A, Guney N, Ozturk S, et al. (2006). Increased sister chromatid exchange frequency in young women with breast cancer and in their first-degree relatives. Cancer Genet. Cytogenet. 171: 65-67. http://dx.doi.org/10.1016/j. cancergencyto.2006.06.005

Çelik DA, Koşar PA, Ozçelik N and Eroğlu E (2013). Cytogenetic finding of breast cancer cases and in their first-degree relatives. J. Breast Cancer 16: 285-290. http://dx.doi.org/10.4048/jbc.2013.16.3.285

Colleoni M, Cole BF, Viale G, Regan MM, et al. (2010). Classical cyclophosphamide, methotrexate, and fluorouracil chemotherapy is more effective in triple-negative, node-negative breast cancer: results from two randomized trials of adjuvant chemoendocrine therapy for node-negative breast cancer. J. Clin. Oncol. 28: 2966-2973. http://dx.doi. org/10.1200/JCO.2009.25.9549

Dhillon VS and Dhillon IK (1998). Chromosome aberrations and sister chromatid exchange studies in patients with prostate cancer: possible evidence of chromosome instability. Cancer Genet. Cytogenet. 100: 143-147. http://dx.doi.org/10.1016/ $\underline{\text { S0165-4608(97)00022-8 }}$

Digkas EN, Chrisafi S, Passadaki T, Tsalkidis A, et al. (2010). In vitro and in vivo cytogenetic effects of recombinant human erythropoietin on the frequency of sister chromatid exchanges alone or in combination with mitomycin C. Chemotherapy 56: 239-247. http://dx.doi.org/10.1159/000316849

Dignam JJ (2001). Efficacy of systemic adjuvant therapy for breast cancer in African-American and Caucasian women. J. Natl. Cancer Inst. Monogr. 2001: 36-43. http://dx.doi.org/10.1093/oxfordjournals.jncimonographs.a003458

Elsendoorn TJ, Weijl NI, Mithoe S, Zwinderman AH, et al. (2001). Chemotherapy-induced chromosomal damage in peripheral blood lymphocytes of cancer patients supplemented with antioxidants or placebo. Mutat. Res. 498: 145-158. http://dx.doi. org/10.1016/S1383-5718(01)00278-9

Ely S and Vioral AN (2007). Breast cancer overview. Plast. Surg. Nurs. 27: 128-133, quiz 134-135. http://dx.doi.org/10.1097/01. PSN.0000290281.48197.ae

Gonzalez-Angulo AM, Morales-Vasquez F and Hortobagyi GN (2007). Overview of resistance to systemic therapy in patients with breast cancer. Adv. Exp. Med. Biol. 608: 1-22. http://dx.doi.org/10.1007/978-0-387-74039-3 1

Han Y, Li Q, Xu BH, Zhang P, et al. (2015). Adjuvant chemotherapy may improve survival of patients with luminal A breast cancer and positive lymph nodes. Genet. Mol. Res. 14: 8563-8573. http://dx.doi.org/10.4238/2015.July.31.4

Kang MH, Genser D and Elmadfa I (1997). Increased sister chromatid exchanges in peripheral lymphocytes of patients with Crohn's disease. Mutat. Res. 381: 141-148. http://dx.doi.org/10.1016/S0027-5107(97)00161-9

Kopjar N, Milas I, Garaj-Vrhovac V and Gamulin M (2007). Cytogenetic outcomes of adjuvant chemotherapy in non-target cells of breast cancer patients. Hum. Exp. Toxicol. 26: 391-399. http://dx.doi.org/10.1177/0960327106076812

Lamberti L, Bigatti Ponzetto P and Ardito G (1983). Cell kinetics and sister-chromatid-exchange frequency in human lymphocytes. Mutat. Res. 120: 193-199. http://dx.doi.org/10.1016/0165-7992(83)90163-X

Mourelatos D (1996). Chromosomes study as predictor of chemoresponse of tumours. Cancer J. 9: 136-141.

Perry P and Wolff S (1974). New Giemsa method for the differential staining of sister chromatids. Nature 251: 156-158. http:// dx.doi.org/10.1038/251156a0

Raposa T and Várkonyi J (1987). The relationship between sister chromatid exchange induction and leukemogenicity of different cytostatics. Cancer Detect. Prev. 10: 141-151.

Santos RA, Teixeira AC, Mayorano MB, Carrara HH, et al. (2010). Basal levels of DNA damage detected by micronuclei and comet assays in untreated breast cancer patients and healthy women. Clin. Exp. Med. 10: 87-92. http://dx.doi. org/10.1007/s10238-009-0079-4

Shet T, Agrawal A, Chinoy R, Havaldar R, et al. (2007). Changes in the tumor grade and biological markers in locally advanced breast cancer after chemotherapy--implications for a pathologist. Breast J. 13: 457-464. http://dx.doi.org/10.1111/j.15244741.2007.00465.x 
Tazzite A, Nadiffi S, Kottwitz D, El Amrani M, et al. (2014). Specific BRCA1 gene variations amongst young Moroccan breast cancer patients. Genet. Mol. Res. 13: 791-798. http://dx.doi.org/10.4238/2014.January.31.5

Tekcan A, Elbistan M and Ulusoy AN (2012). Sister chromatid exchanges in breast cancer patients who underwent chemotherapy. J. Toxicol. Sci. 37: 235-243. http://dx.doi.org/10.2131/jts.37.235

Traoré B, Keita M, Diane S, Dankoro A, et al. (2012). Clinicopathological study of breast diseases presenting to the surgical oncology unit of Donka University Hospital in Conakry, Guinea. West Afr. J. Med. 31: 227-231.

Wang J, Wang T, Yin GY, Yang L, et al. (2015). Glutathione S-transferase polymorphisms influence chemotherapy response and treatment outcome in breast cancer. Genet. Mol. Res. 14: 11126-11132. http://dx.doi.org/10.4238/2015.September.22.6

Xu ZD, Yajima A, Abe Y, Soh K, et al. (1983). Effects of radiotherapy and chemotherapy on sister chromatid exchanges in peripheral lymphocytes of the patients with gynecologic cancer. Tohoku J. Exp. Med. 141: 175-181. http://dx.doi. org/10.1620/tjem.141.175 